\title{
NON-SMALL CELL LUNG CANCER (NSCLC) - A COMPREHENSIVE REVIEW
}

\author{
Rashmi Anupozu*, Manidhar Vanamala \\ Department of Pharm-D, MallaReddy Institute of Pharmaceutical Sciences (MRIPS), \\ Maisammaguda, Hyderabad, Telangana- 500100. \\ Email id-anupozurashmi095@gmail.com
}

\begin{abstract}
Non-Small Cell Lung Cancer (NSCLC) is the most commonly diagnosed type of cancer globally and is the leading cause of cancer death in men and second leading cause of cancer death in women. The frequently seen subtypes of NSCLC in patients are adenocarcinoma, Squamous cell carcinoma and large cell carcinoma. Lung cancer, depending on the stage and kind, it may act differently in each individual. The usual symptoms may include cough, hemoptysis, fatigue, SOB, chest pain, loss of weight etc. and these symptoms differ depending on the site of metastasis. Tobacco smoking is known to the main aggravating risk factor of lung cancer. Other factors like occupational exposure to chemicals and ionizing radiation, passive smoking, genetics, air pollution, diet and alcohol may all contribute in developing of lung cancer in the individuals. Different mechanisms are involved in the development of the tumor in the body. Large number of diagnostic procedures are available to diagnose the lung cancer. Appropriate procedure for diagnosis is selected depending on the site, type, metastasis and size of the tumor. The fundamental basis for the management of cancer is the stage classification. Clinical stage in the patients can be determined by their laboratory results, bronchoscopy findings, imaging tests and by physical examination. Subsequently patients are staged between stage-1 to stage-4. Different types of treatment options are available for treating NSCLC which includes surgery, radiation therapy, chemotherapy, targeted therapy, immunotherapy, palliative care. The main objective of this review is to provide the updated epidemiology, risk factors, diagnostic procedures, staging and treatments for NSCLC.
\end{abstract}

Keywords: Clinical staging, epidemiology, lung cancer, NSCLC, risk factors, symptoms, treatment.

\section{Introduction}

The primary organs of the respiratory system in humans is lungs. The lungs are air-filled organs that are located on each side of the chest. The left lung is slightly small due to asymmetrical placement of heart has two lobes and the right lung has three major lobes. When a person inhales, the oxygen that is present in the air is absorbed by the lungs and this oxygen is brought into the bloodstream for delivery to the rest of the body. The cells of the body uses oxygen and in return releases carbon dioxide which is carried by the bloodstream back to the lungs. This carbon dioxide leaves the body when the person exhales. Lungs are enclosed by a thin tissue layer known as pleura. The lungs are made up of different types of cells, most of which are epithelial cells. The airways are lined by these epithelial cells which makes mucus. This mucus lubricates and protects the lungs. Blood cells, hormone-producing cells, nerve cells and structural or supporting cells are all present in the lungs.

\section{Epidemiology}

The most frequently diagnosed type of cancer globally is the lung cancer. It is the leading cause of death because of cancer in men and the second leading cause of cancer death (after breast cancer) in women. As of statistics in 2018, 1,735,350 new cancer cases and 609,640 cancer deaths are known to be occurred in United States [2]. According to The American Society's estimates in United States for 2019 are 228,150 new cases and 142,670 deaths due to lung cancer are reported [3]. In Indian males and females, lung cancer constitutes about $6.9 \%$ of all new cancer cases and $9.3 \%$ of all cancer related deaths and high incidence is seen in Mizoram in both sexes [5]. Among Indian patients, higher numbers were non-smokers, compared to the west and adenocarcinoma was the most commonly seen subtype [4]. However the incidence and mortality in other places highly differ by the varying trends in smoking patterns.

Types of Lung cancer- [6]

Lung cancer is mainly of two types, small cell lung cancer (SCLC) also called as oat cell cancer due to the resemblance of the cells to oat grains and non-small cell lung cancer (NSCLC). These types are differentiated/diagnosed based on how these cells look under the microscope. Greater than $80 \%$ of lung cancers are of non-small cell type. 


\section{Non-small cell lung cancer (NSCLC):}

This is the most commonly seen type of lung cancer. There are three common types. These subtypes usually start from different types of lung cells but are grouped together as NSCLC because they are all treated in the same way and has similar outlook (prognoses).

- Adenocarcinoma: This type is the most commonly seen. It tends to start in the cells that secrete the mucus and seen mainly in current or former smokers but is most common type of lung cancer in the non-smokers. Adenocarcinoma is found usually in the outer parts of the lung and is seen more frequently in women than in men.

Squamous cell carcinoma: This type tends to start in the squamous cells that line the inside of lung airways. This develops more often in people with history of smoking. It is found to start in the central part of lungs, near a main airway (bronchus).

- Large cell carcinoma: This is very less frequently seen type of NSCLC which tends to grow and spread rapidly that makes it harder to treat and tends to appear in any part of the lung. The cells in this type appear large and round under the microscope.

Other subtypes: A small number of other subtypes of NSCLC include adeno squamous carcinoma and sarcomatoid carcinoma which are less commonly seen.

\section{Small cell lung cancer (SCLC):}

Around $10 \%$ to $15 \%$ of all lung cancers in people are small cell type. This type of cancer tends to grow and spread rapidly than Non-small cell type. At very early stages it spreads to other parts of the body. At the time of patient's diagnoses, $70 \%$ of the cases with SCLC have the cancer that has already spread.

\section{Other type of lung tumors:}

- Lung carcinoid tumors- These type account for less than $5 \%$ of lung tumors. These tend to grow very slowly.

Other lung tumors- Several other types of lung cancer include adenoid cystic carcinomas, lymphomas, sarcomas, benign lung tumors such as hamartomas which are very rare.

- Cancer that spread to lungs- Cancer that primarily begins in other organs such as breast, cervix, pancreas, kidney may sometimes spread (metastasize) to lungs but which are not considered as lung cancers. The treatment is different for these metastatic cancer to lungs and is based on the primary site where it started.

\section{How lung cancer spreads?}

Like all the other cancers, lung cancer, depending on the stage it is in and kind of lung cancer may act differently in each person. Once the lung cancer spreads outside the lungs, it often goes to the same places. The foremost place it spreads to, is the lymph nodes that are present in the center of the chest which are called as mediastinal lymph nodes. It may also spread to lymph nodes that are present in the lower neck. In the later stages, it may spread (metastasize) to distant organs like the liver, brain, bone or adrenal gland.

Signs and symptoms of NSCLC- [7], [8], [9]

Symptoms does not appear in most of the cases until the cancer is quite advanced. The early symptoms maybe a mild cough and shortness of breathe depending on the affected part of lung and may vary for each person. But as the cancer advances the symptoms may become more intense or worsen. Commonly seen symptoms include-

Cough that is constant or gets worse

Coughing up of rust-colored sputum (spit or phlegm) or blood (Hemoptysis)

Loss of appetite

Unexplained loss of weight

Pain in the chest, shoulders or back that may worsen especially when deep breathing, laughing or coughing.

Shortness of breath that may occur during everyday activities

Frequently falling sick due to infections like pneumonia, bronchitis that doesn't go away

or keep coming back

Hoarseness or new onset of wheezing

Fatigue or feeling tired or weak

Pleural effusion (A tumor in the lung may cause fluid to buildup in the lung or the space surrounding the lungs and may cause the lung to collapse) 
Less commonly seen symptoms may include-

Difficulty or pain during swallowing

Swelling in the neck or face

Finger clubbing in some case

In advances stages the cancer metastasizes to distant organs may cause different symptoms. These symptoms depends on the site of spread which may include-

Neurological changes- Lung cancer may metastasize to brain and may cause headaches, nausea, seizures, speech difficulties, numbness or weakness in the arms and legs.

Lumps- Cancer may spread to the lymph nodes and these tumors appear as lumps near the skin surface such as those above the collarbone and in the neck.

Yellowing of eyes and skin (jaundice) which is due to spread of cancer to the liver.

Bone pain (pain in the back and hips) or fractures maybe seen when the cancer spreads to the bone.

\section{Risk Factors}

The risk factors associated with lung cancer are as follows

1. Tobacco smoking [10], [11], [12], [13] - Tobacco smoking, mainly cigarette smoking is the leading cause of lung cancer accounting for about $80-90 \%$. Nicotine is the primary influencer of addiction to tobacco. After nicotine and water have been removed, tar is the total particulate matter of cigarette smoke and exposure to tar is the major component to risk of lung cancer. A low risk of lung cancer is associated with cigar smoking and pipe smoking when compared to the cigarette smoking which is due to the differences in frequency of smoking and depth of inhalation. In India, high risk of lung cancer has been associated with the consumption of local smoked tobacco products like bidi, loose tobacco rolled in leaf and hookah. The relative risk of lung cancer is low in ex-smokers and non-smokers when compared to the smokers. The duration of smoking and number of cigarettes smoked per day influences the rise of risk in lung cancer.

2. Secondhand or Passive smoking [10], [11], [12], [13], [14], [15], [23] - It is a mixture of the smoke that comes from the burning end of a cigarette, cigar, or pipe, and the smoke breathed out by the smoker and which is inhaled the people nearby. This is also called as Environmental tobacco smoke. About 1.6\% of lung cancers are seen in passive smokers. It is estimated that secondhand smoking may cause 3,000 lung cancer deaths per year in the United States and globally about 21,400 deaths per year [14]. A recent report from a study showed that passive smoking during childhood increased the risk of lung cancer by 3.6 fold in adulthood [15]. An established causal relationship between the secondhand smoking and lung cancer are responsible for $1.6 \%$ of lung cancers [23].

3. Never smokers- Never smokers are people who have smoked less than 100 cigarettes in their whole lifetime or have never smoked. It ranks the seventh most common cause of cancer death worldwide, if lung cancer in never smokers is separately considered [17]. Globally, it accounts for about $25 \%$ of all lung cancers approximately 300,000 in women and seen commonly in Asians and African Americans. The subtype adenocarcinoma is more common in non-smokers than the smokers.

4. Occupational exposure- Many different substances at workplaces are known to be carcinogens and are responsible for causing lung cancer. Exposure to tar and soot (benzopyrene) by the workers at the concentrations higher than that present in the urban air are at higher risk of developing lung cancer [12]. Some of the carcinogens known are chromium, arsenic, nickel, radon, asbestos, beryllium, silica, chloromethyl ether, vinyl chloride, diesel exhaust and polycyclic aromatic hydrocarbons. Amongst all the above mentioned carcinogens, asbestos is the most common and widely known occupational cause of lung cancer. Globally, 152,000 deaths and approx. 1.6 million disability-adjusted life years were estimated that are due to occupational carcinogen exposure [18].

5. Diet and Alcohol- Nearly $30 \%$ of all cancers are caused due to diet [19]. It was reported in a prospective survey that diet rich in vegetable, fruits, micronutrients such as carotenoids are known to decrease the risk of lung and other cancers. In response to this, many other trials were conducted later-on. Amongst them, one of the study was The Alpha-Tocopherol, Beta Carotene Cancer Prevention (ATBC) Study but unfortunately the studies reported that in the group of smokers receiving beta-carotene, a high rate of mortality due to lung cancer and heart disease was seen and also among the male smoker population who are on dietary supplementation with alpha-tocopherol and beta-carotene for five to eight years have shown no reduction in the incidence of lung cancer [20]. People consuming 30g/d of alcohol are known to be at a higher risk of lung cancer than those of ex-alcoholics. 
6. Air Pollution- Due to cumulative exposure to the ambient air pollution can cause long term consequences like adverse effects on lung growth, lung cancer and is also known to cause atherosclerosis and asthma [21]. A study stated the biomarkers of exposure to air pollution which are mainly the DNA adducts which are known to increase the risk of lung cancer. An association between the external exposure to air pollution and the increased levels of DNA adducts have been established by many studies [22]. Because of urban air pollution, the attributable proportion of lung cancer is about $11 \%$ in Europe [23].

7. Physical activity and exercises- Physical activity is directly related to the incidence and mortality in site-specific and all-site cancer especially in non-smoking individuals. A study results concluded that incidence of lung cancer is reduced in men by physical activity and reduced mortality in women [24]. Another study data suggests that there is lower risk of lung cancer in physically active individuals [25]. Reduction of $13 \%$ to $30 \%$ in lung cancer risk have been associated with moderate to high levels of leisurephysical activity (LPA) [26].

8. Genetics- As cigarette smoking is the major cause for lung cancer, familial factors also play a major role in the increased risk of lung cancer. Several studies have been done to examine if there is a correlation between the genetic variability in the individuals and lung cancer. Several genomic-wide studies reported that there are three susceptibility loci $(15 \mathrm{p} 25,5 \mathrm{q} 15,6 \mathrm{p} 21)$ on chromosomes which are highly associated with lung cancer family may not contribute to the lung cancer [29]. A family history of lung cancer is associated with increased risk of lung cancer with 1.5 to 4 folds after adjustment for the clustering of smoking in families [30].

9. Exposure to ionizing radiation- Increased risk of lung cancer is seen in people exposed to ionizing radiation. A study concluded that the individuals who have never smoked tobacco presented a reduced risk of lung cancer than the current and ex-smokers who are exposed to radiation. Thus no smoking has benefited people by reducing the lung cancer risk particularly in those exposed to radiation [31]. A pooled analysis of 13-studies in Europe showed that the hazards from residential radon are responsible for $2 \%$ of lung cancers specifically in smokers and recent ex-smokers [32]. There is a higher risk of lung cancer in patients who have gone through previous radiation therapy to chest for other cancers and this risk proportion is high in smokers.

\section{Diagnosis}

A large number of diagnostic procedures are known for diagnosing lung cancer. Depending upon the site, type, metastases and size of tumor, the choice of procedure is selected. Initially minimal invasive methods are more acceptable for diagnosing lung cancer and are the first choice of tests in accessible lymph nodes for confirming the mediastinal diseases. If these needle techniques produce negative results, then these should be followed by surgical biopsy [33]. More invasive method is used later if the above method fails to obtain tissue. Identification of central lesions are more accurate by Transbronchial needle aspiration, Conventional bronchoscopy brushings and washings and sputum cytology. In case of peripheral lesions, can be identified by Computed tomography-guided transthoracic needle aspiration. More effective and safe method to diagnose these peripheral lung lesions is Electromagnetic navigation bronchoscopy (ENB) in combination with PET-CT and rapid on-site cytopathologic examination (ROSE) [34]. Conventional bronchoscopy brushings and washings, Endobronchial ultrasound guided transbronchial needle aspiration (EBUS-TBNA) methods show a lesser sensitivity towards the identification of peripheral lesions. Pleural biopsy can be done to diagnose the pleural effusion in a lung cancer patient [36] or in situations where the findings of pleural fluid cytology are negative [35]. Pleural cytology technique is a minimally invasive procedure which is used to differentiate between the malignant and paramalignant effusion which may alter the stage of the cancer and the treatment approaches [37]. The best diagnostic method for paratracheal, subcranial, and peripheral lymph nodes is the EBUS-TBNA [35] [38]. According to the new International Association for the Study of Lung Cancer (IASLC)/American Thoracic Society (ATS), specifically when searching for genetic abnormalities (like Epidermal growth factor receptor (EGFR) mutations, Anaplastic lymphoma kinase (ALK) translocations), obtaining required tissue material is necessary [39].

\section{Staging}

The most commonly followed staging system for cancer is the Tumor, Node, Metastasis (TNM) staging system which is given by the American Joint Committee on Cancer (AJCC) and the International Union against Cancer (UICC) [40]. The fundamental basis for the management of cancer is the stage classification. T describes the size and extent of the main tumor and if it has grown to the nearby structures or organs. $\mathrm{N}$ describes if the cancer has spread to lymph nodes nearby. M describes if the cancer has spread to distant organs like brain, adrenal glands, bone, high numbers imply that the cancer is more advanced [41]. The two main types of staging in lung cancer are the clinical staging and the pathological staging. Based on the results of the lab tests, physical examination, bronchoscopy findings and imaging tests, clinical stage can be determined. The pathological stage is determined by examination of the removed tissue, only if after the surgery is done. After the T, N and $\mathrm{M}$ categories are described, this particulars are merged to give the tumor an overall stage (I-IV) [40]. In general, 
Stage 1- The cancer is seen only in the lungs and has not spread to any lymph nodes.

Stage 2- The cancer is located in the lungs and in the nearby lymph nodes.

Stage 3-Cancer is found in the lungs and in the lymph nodes located in the middle of the chest which is also known as locally advanced disease.

This stage 3 can be further divided into two stages, IIIA and IIIB.

In stage IIIA, the cancer has spread to only lymph nodes on the same side of the chest where initially the cancer has started.

In stage IIIB, the cancer has spread to lymph nodes to opposite side of the chest.

Stage 4- The cancer has spread to distant organs like brain, liver, adrenal glands, bone and/or to the opposite lung which is called as metastasis [42], [43].

\section{Complications}

In general stage-specific treatment is given in lung cancer patients. Despite aggressive treatment, most of the patients die with 5 years of diagnosis of the cancer. Due to the local complications, there is a decrease in both, quality of life and functional status of the patients. Thus, treating these local complications can immediately and straight away improves the quality of life and functional status of patients. These complications differ in individuals and has to be treated accordingly and collectively through a multi-disciplinary team [44]. Pleural effusion is one such complication which is fluid buildup in the pleura (membrane covering the lungs), which causes breathlessness. Neuropathy is seen in some patients which is due to compression of the nerves by the tumor causing pain, numbness and weakness. Heart complications are seen if the tumor develops near to the major blood vessels. In some patients, the tumor may grow towards the food pipe which makes the patient difficult to swallow. Thus a decrease in the appetite is most commonly seen in the lung cancer patients. This tumor may also grow into the airway and block it which may cause shortness of breath and pneumonia. All these complications are treated in multiple ways as per the health status of the patient [45]. A study conducted by Yasuo Sekine concluded with the obtained data that the post-operative pulmonary complications are common in patients having COPD which may in turn effects the long-term survival of the patient [46].

\section{Treatment}

Non-small cell lung cancer can be treated with various methods like SUREGEY, RADIO- FREQUENCY ABLATION, RADIATION THERAPY, CHEMOTHERAPY,TARGETED THERAPY, IMMUNOTHERAPY and PALLIATIVE PROCEDURES which are used either alone or in combination. Treatment options are chose based on the cancer stage and its extent [47].

Surgery- Surgical removal of the tumor is the primary suggestion for the early stages of includes pneumonectomy (removal of entire cancer infested lung), lobectomy (removal of cancer infested lobe), segmentectomy (removal of cancer infested lobe segment), sleeve resection (removal of tumor only) and videoassisted thoracic surgery (VATS).

VATS procedure is done by a thoracoscope which has a light and a tiny camera connected to monitor which helps in identifying the extraction of cancer tissue with minimal invasion and fewer complications [48].

Although surgery is ideal in NSCLC treatment, but it is still a major operation bearing serious side effects. Thus, it isn't preferred always. Side effects of surgery rely on the extent of surgery and patient's overall health. Effects may include reactions to anesthesia, excess bleeding, blood clots, pneumonia, wound infections and in rare cases, death may be seen.

Radio Frequency Ablation (RFA) - Radio-frequency ablation is technique using radio waves to treat cancer by creating heat. RFA is done by a probe (a needle) kept into the tumor through skin of chest with the help of CT scanner for the accurate positioning. An electrode present in the probe creates radio-frequency energy to produce heat and destroy the cancer tissue. Adverse effects of RFA are pain, fatigue and possible risk of pneumothorax [49].

Radiation Therapy (RT) - RT uses beams of extreme energy to kill cancer cells. In RT X- rays are most often used but sometimes protons or other types of energy beams are also used. The term RT refers external beam radiation. During the treatment, high-energy beams are send from an external machine that aims at precisely marked point on body. RT acts by destroying the genetic material of cell that commands the growth and division [50].

Based on stage of NSCLC and various other factors, RT is used in different methods- as a primary treatment (in case if surgery is not an option), as a pre-surgical procedure, as neo- adjuvant therapy to reduce the size of the tumor or for an easy access, to treat post- surgical remnants of cancer, as adjuvant therapy, sometimes in combination with CT for better treatment outcome, to treat metastatic cancer and as a palliative treatment. 
Evolution in RT have increased the treatment methods to be more accurate with lesser radiation complications.

Methods are as follows:

- Steriostactic Body Radiation Therapy (SBRT): SRBT uses strong beams of high dose radiation for less period of time rather than small dose for longer period. Several beams are targeted from different angles towards tumor. To enhance the precision, special body frames are used to reduce the movement of tumor during breathing.

- 3D Confirmal Radiation Therapy (3D-CRT): This method uses customized computers to map the tumor location precisely, which also shapes the radiation beams aimed at tumor to reduce the damage to noncancerous tissue.

- Intensity Modulated Radiation Therapy (IMRT): This method is similar to 3D-CRT but along with shaping, strength of the beams can also be adjusted in this method.

In few cases brachytherapy is suggested, in which radiation therapy is provided internally meaning a radioactive material in from of small pellets is placed in tumor or next to it [51].

As a matter of fact, during radiation both healthy and cancer cells are damaged. But the goal is to destroy as few healthy cells as possible because the normal cells can repair the damage done by radiation.

Side effects include fatigue, nausea, vomiting, loss of appetite, loss of weight, dermatological problems and hair loss on site of radiation.

Chemotherapy (CT) - Usage of anti-cancer drugs to treat cancer is called as chemotherapy.

Chemotherapy regimen usually includes a certain number of cycles administered over a period of time, but the drugs given are based on the type of cancer being treated.

Drugs most commonly used to treat lung cancer are:

- Carboplatin or cisplatin

- Paclitaxel (taxotere)

- Doxetaxel (taxol)

- Gemcitabine (gemzar)

- Vinorelbine (navelbine)

- Etopside

- Pemetrexed (alimta)

These drugs act on the cell mechanisms responsible for growth and division either directly or indirectly. CT is preferred in various cases like neo-adjuvant chemotherapy, adjuvant chemotherapy, for locally advanced NSCLC and for metastatic stage 4 NSCLC [52].

Chemotherapy is associated with certain side effects like hair loss, mouth sores, loss of appetite, weight changes, nausea, vomiting diarrhea and constipation [53].

CT has shown the improvement in both length and quality of life for patients with lung cancer of all stages.

- Targeted Therapy - Targeted therapy is usage of drugs which act on specific mechanisms that are required for cell growth and gene mutations causing cancer. There are various classes of drugs included in targeted therapy are as follows

$\checkmark$ Angiogenetic inhibitors: Inhibits the angiogenesis (formation of new blood vessels) by targeting vascular endothelial growth factor.

E.g. Bevacizumab, Ramucirumab.

Commonly seen side effects by using angiogenetic inhibitors include high BP, fatigue, low WBC count with increased risk of infections, loss of appetite and bleeding. As the risk of bleeding is high in squamous cell type of NSCLC, usage is not recommended.

$\checkmark$ EGFR inhibitors: Acts by inhibiting epidermal growth factor receptor (EGFR) is a surface protein involves in growth and division of cell, which mutation leads to cancer. E.g. Erlotinib, Afatnib, Geftinib, Dacomitinib.

Side effects seen commonly by using EGFR inhibitors are diarrhea, mouth sores, loss of appetite, skin problems sometimes leading to an infection.

$\checkmark$ Drugs targeting ALK gene mutations: ALK gene mutation lead to production of abnormal ALK protein causing cell to grow and spread abnormally.

E.g. Crizotinib, loratinib, ceritinib [54]. 
Possible side effects caused are nausea, vomiting, diarrhea, constipation, fatigue, changes in vision, rarely inflammation in lungs liver and nerve damage.

- Immunotherapy - Immunotherapy activates the immune system to recognize and kill cancer cells more effectively by acting on certain proteins responsible for immune reactions like PD-1 \& PD-L1 which are kept away from attacking the cell of body.

E.g. Nivolumab, pembrolizumab, atezolizumab.

Adverse effects of immunotherapy includes fatigue, cough, nausea, diarrhea, loss of appetite, skin rash and less often autoimmune reactions and infusion reactions [55].

\section{- Palliative Treatment -}

Palliative treatment is a concept in medical care aiming to identify and address the physical, psychological, spiritual and practical burdens of illness required in life-threatening diseases like cancer. Palliative treatment includes

$\checkmark \quad$ Palliative symptom management starts with a small talk between patient and provider assessing the symptoms and providing required information about possible treatment options.

$\checkmark \quad$ Palliative surgical procedures are required in order to treat complications by lung cancer like airway obstruction, hemoptysis, pleural effusion which can be treated by bronchoscopy methods and thoracocentesis.

$\checkmark$ Palliative chemotherapy is a non-curative chemotherapy primarily aiming to relieve the symptoms.

$\checkmark \quad$ Palliative radiation can alleviate pain and neurologic drawbacks from brain and spinal metastasis \& prevention of impending pathologic fracture.

Interference such as breathing techniques, imagery guidance can have a positive impact on psychological symptoms such as anxiety \& depression. The social, spiritual support for patients and families shows impact on physical symptoms as well as quality of life [56].

\section{Treatment for NSCLC based on stage: [57]}

Based on different stage of NSCLC different treatment methods may be recommended.

\section{- $\quad$ Stage 1 and 2 NSCLC:}

Primarily, surgery is the widely preferred to treat stage 1 and 2, but in some cases for example- patients with large size of tumor or with the signs of metastasis in lymph nodes shown, chemotherapy is beneficial. Chemotherapy is provided as both neo-adjuvant CT and adjuvant CT. Adjuvant CT with cisplatin is recommended for stage 2 cancer surgically removed. Occasionally radiotherapy is also preferred in cases where surgery is not an option.

\section{- $\quad$ Stage 3 NSCLC:}

Treatment plan for stage 3 NSCLC is based on tumor location, size and the involved lymph nodes.

Stage 3A NSCLC treatment plan may include combination of RT, CT \&/or surgery. CT \& RT are given together called as concurrent chemoradiation therapy or one after the other called as sequential chemoradiation therapy. Sometimes patient may not be able to tolerate chemotherapy, radiation or surgery, then immunotherapy is preferred.

Stage 3B NSCLC tumor might have spread to farther lymph nodes or to other parts in chest so surgery is not an option. In such cases chemoradiation or immunotherapy is recommended.

Stage 4 NSCLC:

Stage 4 NSCLC is also considered as metastatic NSCLC which means the cancer is widely spread to other parts of body varied from origin. Treatment plan will be based on metastasis. If cancer is spread to only one other site, surgery might help along with CT, RT and palliative care. In case of multiple metastatic sites, treatment plan may rely on gene mutations, treated with Targeted therapy along with CT and palliative procedures.

Nil

\section{Acknowledgement-}

\section{Conflicts of interest-}

Authors declare no conflicts of interest. 


\section{REFERENCES:}

[1] Melissa Conrad Stoppler. Lung Anatomy: How the lungs work. Available on: https://www.medicinenet.com/lungs_design_and_purpose/article.htm\#human_lung_an atomy_definition_and_facts

[2] Siegel RL, Miller KD. Jemal A (2018) cancer statistics. Ca Cancer J Clin. 2018, 68(1): 7-30.

[3] Key statistics for lung cancer. The American Cancer Society. Available on: https://www.cancer.org/cancer/lung-cancer/about/keystatistics.html

[4] Noronha V, Dikshit R, Raut N, Joshi A, Pramesh CS, George K, Agarwal JP, Munshi A, Prabhash K. Epidemiology of lung cancer in India: Focus on the differences between non-smokers and smokers: A single-centre experience. Indian journal of cancer. 2012, 49(1):74.

[5] Malik PS, Raina V. Lung cancer: Prevalent trends \& emerging concepts. The Indian journal of medical research. 2015, 141(1):5.

[6] Zheng M. Classification and pathology of lung cancer. Surgical Oncology Clinics. 2016, 25(3):447-68.

[7] Collins LG, Haines C, Perkel R, Enck RE. Lung cancer: diagnosis and management. American family physician. 2007, 75(1):56-63.

[8] Signs and symptoms of lung cancer. The American Cancer Society. Available on: https://www.cancer.org/cancer/lungcancer/detection-diagnosis-staging/signs- symptoms.html

[9] Bjerager M, Palshof T, Dahl R, Vedsted P, Olesen F. Delay in diagnosis of lung cancer in general practice. Br J Gen Pract. 2006, 56(532):863-8

[10] Malhotra J, Malvezzi M, Negri E, La Vecchia C, Boffetta P. Risk factors for lung cancer worldwide. European Respiratory Journal. 2016, 48(3):889-902.

[11] Cruz CS, Tanoue LT, Matthay RA. Lung cancer: epidemiology, etiology, and prevention. Clinics in chest medicine. 2011, 32(4):60544

[12] Alberg AJ, Brock MV, Ford JG, Samet JM, Spivack SD. Epidemiology of lung cancer: Diagnosis and management of lung cancer: American College of Chest Physicians evidence-based clinical practice guidelines. Chest. 2013, 143(5):e1S-29S.

[13] Molina JR, Yang P, Cassivi SD, Schild SE, Adjei AA. Non-small cell lung cancer: epidemiology, risk factors, treatment, and survivorship. InMayo Clinic Proceedings 2008 May 1 (Vol. 83, No. 5, pp. 584-594). Elsevier.

[14] Öberg M, Jaakkola MS, Woodward A, Peruga A, Prüss-Ustün A. Worldwide burden of disease from exposure to second-hand smoke: a retrospective analysis of data from 192 countries. The lancet. 2011, 377(9760):139-46.

[15] Vineis P, Airoldi L, Veglia F, Olgiati L, Pastorelli R, Autrup H, et al. Environmental tobacco smoke and risk of respiratory cancer and chronic obstructive pulmonary disease in former smokers and never smokers in the EPIC prospective study. Bmj. 2005, 330(7486):277.

[16] Sun S, Schiller JH, Gazdar AF. Lung cancer in never smokers - a different disease. Nature Reviews Cancer. 2007, 7(10):778-90.

[17] Sun S, Schiller JH, Gazdar AF. Lung cancer in never smokers-a different disease. Nature Reviews Cancer. 2007, 7(10):778-90.

[18] Driscoll T, Nelson DI, Steenland K, Leigh J, Concha-Barrientos M, Fingerhut M, Prüss- Üstün A. The global burden of disease due to occupational carcinogens. American journal of industrial medicine. 2005, 48(6):419-31.

[19] Willett WC, Trichopoulos D. Nutrition and cancer: a summary of the evidence. Cancer Causes \& Control. 1996,178-80.

[20] Alpha-Tocopherol Beta Carotene Cancer Prevention Study Group. The effect of vitamin E and beta carotene on the incidence of lung cancer and other cancers in male smokers. New England Journal of Medicine. 1994, 330(15):1029-35.

[21] Künzli N, Tager IB. Air pollution: from lung to heart. Swiss Med Wkly. 2005, 135(47-48):697-702.

[22] Vineis P, Husgafvel-Pursiainen K. Air pollution and cancer: biomarker studies in human populations. Carcinogenesis. 2005, 26(11):184655.

[23] Boffetta P. Human cancer from environmental pollutants: the epidemiological evidence. Mutation Research/Genetic Toxicology and Environmental Mutagenesis. 2006, 608(2):157-62.

[24] Alfano CM, Klesges RC, Murray DM, Bowen DJ, McTiernan A, Vander Weg MW, et al. Physical activity in relation to all-site and lung cancer incidence and mortality in current and former smokers. Cancer Epidemiology and Prevention Biomarkers. 2004, 13(12):2233-41.

[25] Lee IM. Physical activity and cancer prevention-data from epidemiologic studies. Medicine \& Science in Sports \& Exercise 2003, 35(11):1823-7.

[26] Molina JR, Yang P, Cassivi SD, Schild SE, Adjei AA. Non-small cell lung cancer: epidemiology, risk factors, treatment, and survivorship. InMayo Clinic Proceedings 2008 May 1 (Vol. 83, No. 5, pp. 584-594). Elsevier.

[27] Brennan P, Hainaut P, Boffetta P. Genetics of lung-cancer susceptibility. The lancet oncology. 2011, 12(4):399-408.

[28] Ankathil R. Tobacco, genetic susceptibility and lung cancer. Tobacco Use Insights. 2010 Jan;3:TUI-S2819.

[29] Bermejo JL, Hemminki K. Familial lung cancer and aggregation of smoking habits: a simulation of the effect of shared environmental factors on the familial risk of cancer. Cancer Epidemiology and Prevention Biomarkers. 2005, 14(7):1738-40.

[30] Schwartz AG, Bailey-Wilson JE, Amos CI. Genetic susceptibility to lung cancer. InIASLC Thoracic Oncology 2018 Jan 1 (pp. 46-51).

[31] Sauvaget C, Nishi N, Soda M. Lifestyle, Ionizing Radiation Exposure and Lung Cancer Risk. Epidemiology. $2006,17(6): S 531$.

[32] Malhotra J, Malvezzi M, Negri E, La Vecchia C, Boffetta P. Risk factors for lung cancer worldwide. European Respiratory Journal. 2016, 48(3):889-902.

[33] Silvestri GA, Gonzalez AV, Jantz MA, Margolis ML, Gould MK, Tanoue LT, et al. Methods for staging non-small cell lung cancer: diagnosis and management of lung cancer: American College of Chest Physicians evidence-based clinical practice guidelines. Chest. 2013, 143(5):e211S-50S.

[34] Lamprecht B, Porsch P, Pirich C, Studnicka M. Electromagnetic navigation bronchoscopy in combination with PET-CT and rapid onsite cytopathologic examination for diagnosis of peripheral lung lesions. Lung. 2009, 187(1):55.

[35] Latimer K, Mott T. Lung cancer: diagnosis, treatment principles, and screening. American family physician. 2015, 91(4):250-6.

[36] Rivera MP, Mehta AC, Wahidi MM. Establishing the diagnosis of lung cancer: diagnosis and management of lung cancer: American College of Chest Physicians evidence-based clinical practice guidelines. Chest. 2013, 143(5):e142S-65S.

[37] Rivera MP, Mehta AC, Wahidi MM. Establishing the diagnosis of lung cancer: diagnosis and management of lung cancer: American College of Chest Physicians evidence-based clinical practice guidelines. Chest. 2013, 143(5):e142S-65S.

[38] Herth FJ, Eberhardt R, Vilmann P, Krasnik M, Ernst A. Real-time endobronchial ultrasound guided transbronchial needle aspiration for sampling mediastinal lymph nodes. Thorax. 2006, 61(9):795-8.

[39] W.D. Travis, E. Brambilla, P. Van Schil, G.V. Scagliotti, R.M. Huber, J-P. Sculier, et al. European Respiratory Journal 2011, 38: 239243.

[40] Tsim S, O’Dowd CA, Milroy R, Davidson S. Staging of non-small cell lung cancer (NSCLC): a review. Respiratory medicine. 2010, 104(12):1767-74.

[41] American cancer society. Non-small cell lung cancer stages, Available from https://www.cancer.org/cancer/lung-cancer/detectiondiagnosis-staging/staging- nsclc.html

42] A program of

care. Types and staging of lung cancer, Available

in 
https://www.lungcancer.org/find_information/publications/163-lung_cancer_101/268-types_and_staging\#menu

[43] Healthline. Everything you need to know about lung cancer, Available from https://www.healthline.com/health/lung-cancer

[44] Sonett JR. Local complications of non-small-cell lung cancer. Current treatment options in oncology. 2002, 3(1):59-65.

[45] MEDIACAL NEWS TODAY. Lung cancer complications: what you should know, Available from https://www.medicalnewstoday.com/articles/316503

[46] Sekine Y, Behnia M, Fujisawa T. Impact of COPD on pulmonary complications and on long-term survival of patients undergoing surgery for NSCLC. Lung Cancer. 2002, 37(1):95-101.

[47] A programme of cancer care, Non-small lung cancer treatment, Available from https://www.lungcancer.org/find_information/publications/163-lung_cancer_101/269-non_small_cell_lung_cancer_treatment

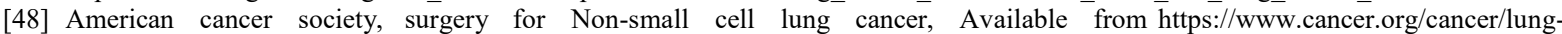
cancer/treating-non-small-cell/surgery.html

[49] Cancer research UK, Radio-frequency ablation, Available from https://www.cancerresearchuk.org/about-cancer/lungcancer/treatment/radiofrequencyablation-rfa

[50] Mayo clinic, Radiation therapy, Available from https://www.mayoclinic.org/testsprocedures/radiation-therapy/about/pac-20385162

[51] American cancer society, Radiation therapy for Non-small cell lung cancer, Available fromhttps://www.cancer.org/cancer/lungcancer/treating-non-small- cell/radiationtherapy.html

[52] Lung cancer - Non-small cell : Types of treatment, Available from https://www.cancer.net/cancer-types/lung-cancer-non-smallcell/types-treatment

[53] American cancer society, Chemotherapy for Non-small cell lung cancer, Available from https://www.cancer.org/cancer/lungcancer/treating-non-small-cell/chemotherapy.html

[54] American cancer society, Targeted therapy for Non-small cell lung cancer, Available from https://www.cancer.org/cancer/lungcancer/treating-non-small- cell/targetedtherapies.html

[55] American cancer society, Immunotherapy for Non-small cell lung cancer, Available from https://www.cancer.org/cancer/lungcancer/treating-non-small- cell/immunotherapy.html

[56] Ferrell B, Koczywas M, Grannis F, Harrington A. Palliative care in lung cancer.Surgical Clinics. 2011, 91(2):403-17.

[57] Cancer.Net Editorial Board, lung cancer- Non small cell: Types of treatment, May 2020. 